\title{
Journal to Wiki publication (J2W)
}

Public Domain

\section{Source}

Open Research Glossary

Copying text from a published paper to a wiki (such as Wikipedia or Wikibooks), with attribution: legally possible if the licence of the paper is less restrictive than the licence of the wiki. 